\title{
Introduction aux finances et à la fiscalité de Philippe le Bon dans les années 1420
}

Takemi Kanao

\section{(2) OpenEdition}

1 Journals

Édition électronique

URL : https://journals.openedition.org/cem/952

DOI : $10.4000 /$ cem.952

ISSN : 1954-3093

Éditeur

Centre d'études médiévales Saint-Germain d'Auxerre

Édition imprimée

Date de publication : 15 août 2004

ISSN : 1623-5770

Référence électronique

Takemi Kanao, « Introduction aux finances et à la fiscalité de Philippe le Bon dans les années 1420 », Bulletin du centre d'études médiévales d'Auxerre | BUCEMA [En ligne], 8 | 2004, mis en ligne le 14 mars 2007, consulté le 22 septembre 2022. URL : http://journals.openedition.org/cem/952 ; DOI : https:// doi.org/10.4000/cem.952

Ce document a été généré automatiquement le 22 septembre 2022.

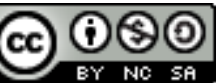

Creative Commons - Attribution - Pas d'Utilisation Commerciale - Partage dans les Mêmes Conditions 4.0 International - CC BY-NC-SA 4.0

https://creativecommons.org/licenses/by-nc-sa/4.0/ 


\title{
Introduction aux finances et à la fiscalité de Philippe le Bon dans les années 1420
}

\author{
Takemi Kanao
}

1 Mes études sur la cour de Bourgogne, notamment sur les messagers et l'organisation des messageries ${ }^{1}$, m'ont conduit à m'intéresser, plus récemment, au fonctionnement des finances et de la fiscalité ducale. Entre avril 2003 et mars 2004, lors d'un séjour à Dijon, j'ai pu consulter les archives bourguignonnes et donner suite à mes recherches, dont on trouvera ici le compte-rendu, avec les perspectives générales de l'enquête.

Pourquoi la Bourgogne et les années 1420 ?

2 Dès les origines, Philippe le Bon cherche à développer sa domination dans les PaysBas. Cette intention ne change pas pendant toute la durée de son règne. Un tel basculement politico-diplomatique s'est accompagné d'une modification sensible de l'administration de sa principauté : l'importance relative du duché et du comté de Bourgogne diminue par rapport aux pays septentrionaux. Philippe gère les pays du Nord, mais ne dirige pas lui-même le gouvernement des pays méridionaux. D'ailleurs, il visite très rarement la Bourgogne. Assurée de son autonomie, elle est bien gouvernée sans contrainte directe du duc et sa contribution financière est continue et régulière. La Bourgogne demeure une source abondante et stable de financement en cas de nécessité. Si des ressources financières importantes assurées par l'activité économique sont la condition indispensable du pouvoir, il est préférable d'analyser la fiscalité de la Bourgogne à celle des pays du Nord, situés au cœur des conflits, pour connaître l'état ordinaire des finances de Philippe le Bon. C'est l'objectif que nous nous sommes fixé pour compléter nos recherches sur la Bourgogne des années 1420. Toutefois, comme cette époque est marquée par une violente fluctuation de la monnaie, nous avons établi une méthode particulière pour convertir et unifier plusieurs monnaies de compte selon la manipulation des receveurs de l'époque.

Aspect global de ses finances 
3 À la suite d'analyses précises des documents financiers de Philippe le Hardi, produits entre 1390 et 1400, Van Nieuwenhuysen conclut que les recettes ordinaires se montaient à $60 \%$ et les recettes extraordinaires à $40 \%$ du total. Le terme "ordinaire" signifie fixé et annuel, c'est-à-dire l'impôt de la production et de la circulation : cens en nature ou en espèce, péage, huitième du vin. En revanche, "extraordinaire" correspond aux recettes sporadiques ou irrégulières : dons et pensions de la part du roi, emprunts directs et aides ducales qui nécessitent l'accord des États. Peut-on appliquer cette conclusion à la structure des finances de Philippe le Bon pour les années 1420 ? Nos recherches rapides sur les recettes générales de "Toutes les Finances" du duc ne permettent pas de conclure. Suivant notre analyse des Recettes générales de Bourgogne, le revenu total varie de 97 mille à 139 mille livres tournois de bonne monnaie. La plupart est constituée par la recette "ordinaire" versée par les receveurs attachés aux bailliages et par les châtelains dans le duché et le comté de Bourgogne, soit entre 51 et 85 mille $£ t$ de bonne monnaie. L'autre partie est suppléée par les aides ducales ou par le "seigneurage" du monnayage. Ainsi, c'est le revenu du monnayage, entre autres, qui remplace le don royal et occupe une place de première importance dans le revenu de Philippe le Bon, en 1420 et 1421. Il faut donc étudier la fiscalité des bailliages de Bourgogne et dépouiller les comptes particuliers du monnayage pour définir l'état du revenu ordinaire du duc.

Les recettes des bailliages et des châtellenies

4 Nous avons dépouillé la très grande masse des documents comptables de la série B des Archives départementales de la Côte-d'Or (Dijon), ce qui représente tout de même pour les seules années 1420 , plus de 130 registres, soit environ cinq mille folios, pour cinq bailliages du duché de Bourgogne : Autun, Auxois, Chalon-sur-Saône, Châtillon-surSeine et Dijon. Chaque bailliage a sa tradition fiscale. L'Autunois se distingue par la "viairie", unité fiscale, non repérée ailleurs. Jean Pucelle, receveur au bailliage, précise et justifie l'augmentation ou la diminution du montant des impôts, qui sont de plusieurs sortes : cens, bien sûr, dixième des denrées et huitième du vin. On y trouve souvent la description des "coutumes" ou l'imposition traditionnelle depuis l'établissement du ban. L'Auxois, en revanche, semble moins intéressant, mais un compte isolé et inclassable, témoignage de la levée d'argent massif, fait réfléchir à l'essence de l'ancienne monnaie. Les recettes et dépenses du Châtillonais attestent d'énormes frais engagés pour un château fort, difficile à maintenir sans aide financière de la part des autres bailliages. Chalon-sur-Saône, connu pour sa foire qui a lieu deux fois par an (été et hiver), témoigne que l'activité commerciale est une source de revenus plus importante que la production agricole. Les marchands paient selon la profession et la largeur du magasin : "loge", "buffet", "étal" et "place”. L'importance de la foire se voit aussi dans le montant élevé du "tabelletinage".

Contre toute attente, il est difficile de caractériser le bailliage de Dijon. Sa contribution aux recettes générales, parfois des milliers de livres, surpasse son revenu total, qui demeure médiocre et assez faible par rapport aux autres bailliages. Est-ce le destin paradoxal du cœur de l'administration? Néanmoins, l'agglomération dijonnaise, qui comprend plusieurs châtellenies aux alentours (Chenôve, Rouvres, Talant...) accuse naturellement sa position non négligeable. Si on y ajoute Nuits et Beaune, la prospérité semble indéniable, principalement en raison de la viticulture. Les comptes de Chenôve nous donnent l'impression qu'ils ont été rédigés spécifiquement pour enregistrer les recettes et les dépenses du clos du duc et construire une partie de l'unité fiscale de 
Dijon. Les revenus de Nuits et Beaune, simples châtellenies, atteignent le niveau médiocre du revenu du bailliage et le dépasse parfois. La description des vendanges y est vive et imagée, même si elle reste comptable : cri annonçant le début des vendanges, recrutement des travailleurs, programme de la journée, achat du froment pour faire cuire du pain et du flan et don de fromage.

Dans le comté de Charolais, le duc place un bailli et un receveur comme dans les autres bailliages de Bourgogne. C'est Jean Pucelle, avant son déplacement à Autun en 1421, qui est receveur et semble établir la comptabilité du bailliage. Lui et ses successeurs suivent fidèlement la directive publiée par la Chambre des comptes et précisent le taux de conversion monnayable du cens fixé et levé en nature (froment, seigle, avoine et cire) et le calcul entre plusieurs monnaies réelles ou fictives.

7 Toutefois, un problème reste pendant : la classification et la partition des impôts. Dans beaucoup de cas, le receveur chargé de lever et d'enregistrer les impôts "ordinaires" ne s'occupe pas de ceux de la gruerie, réservés à un autre receveur. La formation historique de la partition de ces deux unités fiscales n'est pas encore suffisamment expliquée.

Politique et ouvrage de la monnaie

8 La monnaie n'est pas approvisionnée au même rythme. C'est l'ordonnance du roi et du duc qui définit la qualité des pièces d'or et d'argent (nom, caractères et armes de la pièce, titre, taille au marc et cours), désigne les ateliers de fabrication (Dijon, Auxonne, Chalon, Saint-Laurent-lez-Chalon et Cuisery), leur volume de production et précise enfin la date de sortie. En revanche, les comptes du "monnoyage" ou de l'atelier monétaire décrivent le mode de fabrication. Par exemple, les artisans mettent une pièce dans la "boîte" par mille pour la fabrication du "gros" (pièce d'argent), et une pour deux cents pièces pour l'"écu" (pièce d'or). C'est un moyen efficace pour vérifier le nombre des pièces fabriquées et calculer le volume de métal employé. La part de profit du duc, ou "seigneurage" du monnayage, dépend du marc du métal pur, tandis que la part du monnayeur, ou "bressage", est définie suivant le volume du "marc d'œuvre". Ainsi, le monnayage semble une affaire métallurgique. Le duc, les conseillers et les monnayeurs généraux et particuliers s'attendent à profiter d'un cycle pour acquérir des matières premières, les transformer en pièce normalisée et les vendre aux marchés. Ils s'inquiètent du prix d'achat des matériaux plutôt que de celui de revente après la sortie de l'atelier monétaire. L'ordonnance règle donc la position et l'activité du changeur et du marchand de métal au titre du fournisseur des matières premières.

L'administration financière et la Chambre des comptes

9 Émanant de la Chambre du conseil, la Chambre des comptes est établie pour l'audition des comptes de plusieurs "officiers", mais devient peu à peu un organisme central de l'administration financière. Elle parait dès lors comme un centre où convergent toutes les informations plutôt qu'un service indépendant des autres sections administratives des finances, de la fiscalité et de la monnaie. Elle est composée d'un groupe de spécialistes des finances chargé de conseiller le trésorier-gouverneur dans la politique à conduire. Les maîtres ou "gens" de la Chambre des comptes ont une réunion régulière sur toutes les finances du duc avec le trésorier-gouverneur, le maître général de la monnaie de Bourgogne et le receveur général de Bourgogne et, pour les revenus ordinaires du bailliage et de la gruerie ou pour les aides, avec les receveurs particuliers. Les finances du duc ne semblent donc pas gérées par l'ensemble hiérarchisé des "officiers", mais par un groupe dont les membres sont bien informés des problèmes auxquels ils doivent faire 
face. Cette entité construit un réseau de collaboration dont le centre est naturellement occupé par les maîtres de la Chambre des comptes.

Il ne faut pas oublier que les receveurs, dès qu'ils sont chargés d'un office, prêtent serment à la Chambre des comptes devant les maîtres et déposent en même temps une caution dont le montant est en général de mille livres tournois. Ils ne sont donc jamais des commissaires mais des officiers au sens traditionnel. Ainsi, peuvent-ils profiter de leur office comme une source de revenus fructueux. Il en est de même pour les baillis et maîtres particuliers.

Contribution aux recherches archéologiques

Les documents comptables du duc de Bourgogne sont une source de première importance pour des travaux archéologiques, puisqu'ils mentionnent l'enregistrement des frais pour la construction et la réparation des fortifications. Par exemple, les comptes du bailliage de Châtillon-sur-Seine détaillent la réparation du vieux château : achat des matériaux, frais de transport et coût des journées des ouvriers. Même s'ils ne détaillent pas le projet global, la description chiffrée montre l'importance des travaux.

\section{BIBLIOGRAPHIE}

Bibliographie sélective

Finances et fiscalité du duc de Bourgogne

ARNOULD (M.), “Une estimation des revenus et des dépenses de Philippe le Bon en 1445”, dans

Recherches sur l'histoire des finances publiques en Belgique, t. III, Bruxelles, 1974, p. 131-219.

DUBoIS (H.), "Naissance de la fiscalité dans un état princier au Moyen Âge : l'exemple de la Bourgogne”, dans J.-P. GENET et M. LE MÈNE, Genèse de l'État moderne. Prélèvement et redistribution, Paris, 1987.

CONTAMINe (P.), KeRHERVÉ (J.) et RigAudière (A.), sous la dir., L'impôt au Moyen Âge, Paris, 2002. KANAO (T.), "Les finances de Philippe le Bon, duc de Bourgogne de la Maison de Valois ${ }^{1}$. Structure macroéconomique de ses revenus dans les années 1420", The Journal of Kawamura Gakuen Woman's University, 9-1, 1998, p. 39-75.

RAUZIER (J.), Finances et Gestion d'une principauté au XIVe siècle. Le duché de Bourgogne de Philippe le Hardi (1364-1384), Paris, 1996.

SORNAY (J.), "Les États prévisionnels des finances ducales au temps de Philippe le Bon", dans $109^{e}$ Congrès national des sociétés savantes, Dijon 1984, Paris, 1987, p. 35-94.

VAn Nieunenhuysen (A.), Les finances du duc de Bourgogne, Philippe le Hardi (1384-1404). Le montant des ressources, Bruxelles, 1990.

MENJOT (D.) et SANCHEZ MARTINEZ (M.), sous la dir., La fiscalité de villes au Moyen Âge, 3 vol., Paris, 1996-2002. 


\section{Monnaie et système monétaire}

BOMPAire (M.) et DUMAS (F.), Numismatique médiévale, Turnhout, 2000 (coll. "L'atelier du médiéviste", 7).

BonNEviot (M.), "Les changeurs de la Rue au Change [à Chalon-sur-Saône]", Mémoire de la Société d'histoire et d'archéologie de Chalon-sur-Saône, 67, 1998, p. 81-90.

DAY (J.), "Les frappes de monnaies en France et en Europe aux XIV et XV siècles”, dans G. DEPEYROT, T. HACKENS, G. MOUCHARTE, sous la dir., Rythmes de la production monétaire de l'Antiquité à nos jours, Louvain-la-Neuve, 1987, p. 537-578 (coll. "Numismatica Lovaniensia", 7).

DAY (J.), Monnaies et marchés au Moyen Âge, Paris, 1994.

DUMAS-Dubourg (F.), Le monnayage des ducs de Bourgogne, Louvain-la-Neuve, 1988 (coll. "Numismatica Lovaniensia", 8).

GOLDEMAN (P.), "Les changeurs de Bourgogne au XV siècle. Groupe social ou équipe professionnelle ?", dans Actes du $57^{e}$ congrès annuel de la Fédération des sociétés savantes du centre de la France (Bulletin de la Société scientifique, historique et archéologique de la Corrèze, 120, 1998, p. 139-143).

GUERREAU (A.), "Réflexions sur les mutations monétaires en France, à la fin du Moyen Âge", dans G. DEPEYROT, T. HACKENS, G. MOUCHARTE, sous la dir., Rythmes de la production monétaire de l'Antiquité à nos jours, Louvain-la-Neuve, 1987, p. 521-535.

LARDIN (P.), “La crise monétaire de 1420-1422 en Normandie”, dans L'argent au Moyen Âge, Paris, 1998, p. 102-143.

MISKIMIN (H. A.), Money and Power in Fifteenth-Century France, Londres, 1984.

Chambre des comptes et administration financière

contamine (P.) et MAteoni (O.), sous la dir., La France des Principautés : les chambres des comptes, $X I V^{e}-X V^{e}$ siècles, Paris, 1996.

PAViot (J.) et Verger (J.), sous la dir., Guerre, pouvoir et noblesse au Moyen Âge. Mélanges en l'honneur de Philippe Contamine, Paris, 2000.

RIGAULT (J.), "De la Chambre des comptes des ducs de Bourgogne à la Chambre régionale des comptes : une résurrection", dans De la Province à la Région et de la France à l'Europe, 1991, p. 199-202.

\section{NOTES}

1.Voir en français, entre autres, T. KANAO, Les messagers à la cour de Bourgogne à la fin du Moyen Âge, Lille, 1992, "L'organisation et l'enregistrement des messageries du duc de Bourgogne dans les années 1420", Revue du Nord, n. 305, a. LXXVI, 1994, p. 275-297, et "Les Messagers du duc de Bourgogne au début du XV siècle", Journal of Medieval History, vol. 21, 1995, p. 195-226. 
INDEX

Mots-clés : finances, Philippe le Bon, fiscalité 